\title{
It's good to get out
}

\author{
Although the expense involved and pressures of time sometimes make visiting conferences \\ difficult, the benefits are often highly worthwhile.
}

As the conference summer season gets underway (the annual CLEO event in the USA is taking place as this issue goes to press) it seems an apt time to reflect on the benefits of attending such gatherings. The opportunity to present your latest results and hear news from others in the same field is often a strong motivation, but there are often other hidden or unexpected benefits that perhaps get overlooked.

In particular, the opportunity to actually speak to researchers, and not just those working in your own field, can often serve as a very valuable source of inspiration. Although e-mail, websites and $\mathrm{ftp}$ are of course convenient and increasingly popular forms of communication, it should not be forgotten that face-to-face meetings and networking at conferences can prove very thought provoking and help to generate new ideas.

Indeed, these comments apply not just to researchers, but also journal editors, who often attend events as a means to help formulate new ideas for review articles, commentaries, themed issues, and in general to keep up to date with the latest developments. The heart of the Nature Photonics submission and peer-review process is of course electronic, but we always enjoy getting out and speaking to researchers personally about their latest research and hearing opinions as to how we could improve the journal.

For those looking for their next job, be it a postdoc position after finishing a $\mathrm{PhD}$ or a more senior research post, conferences can be a good place to pick up, by word of mouth, the latest job opportunities at universities, research institutions and government or industry labs. Indeed some events even have dedicated career fairs or centres purely

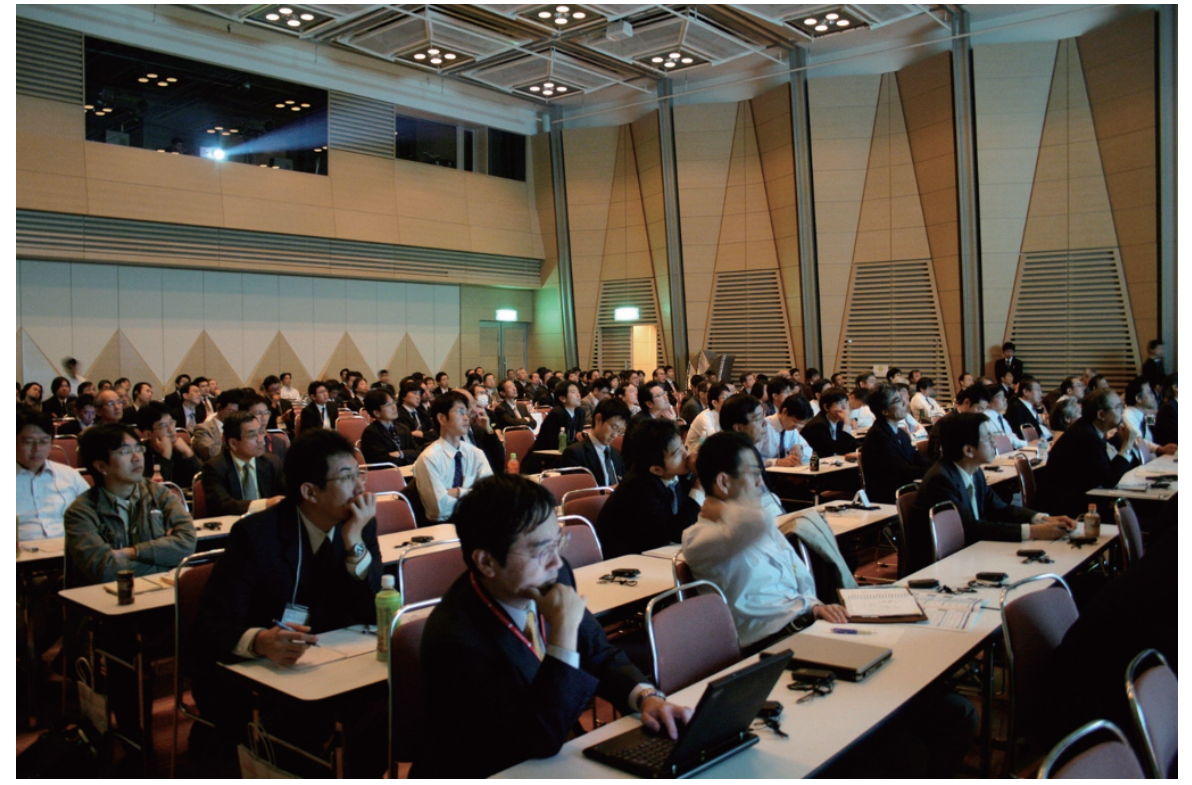

to support the purpose of recruitment. For those considering a move into the commercial world, conferences with an exhibit offer an opportunity to speak to a large number of companies in a convenient fashion to find out if they are hiring and what types of posts they have on offer.

Another benefit of attending events, especially the larger ones with a broader scope, is the ability to gain a 'big picture' overview of what is happening in photonics in general. Many scientists are naturally experts in their own line of research, but they may not be aware of recent breakthroughs in other areas. Given the highly transferable nature of photonics, hearing about the latest developments in different fields can prove extremely useful. It can help with inspiring new ideas for projects, discovering alternative techniques and technologies that prove to be ideal solutions for problems you are facing, or even lead to potential collaborations that would never happen if you stayed in the lab.

Given the large scale of some of the well established conferences in photonics, it can sometimes be hard deciding how to get the most out of them. We find that attending plenary talks, post-deadline sessions and the reception is often high on our list. This combination offers the possibility of receiving accessible overviews of a topic, a round-up of some very recent findings that have been deemed highly important and timeworthy, and of course the chance to do some serious talking. 